\title{
Mortality and associated factors of patients with extensive drug-resistant tuberculosis: an emerging public health crisis in China
}

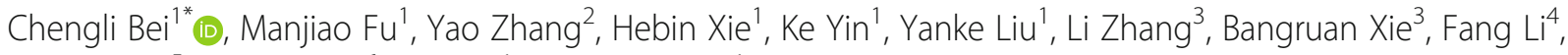
Hua Huang ${ }^{5}$, Yuhong Liu', Li Yang ${ }^{1}$ and Jing Zhou'

\begin{abstract}
Background: Limited treatment options of extensive drug-resistant tuberculosis (XDR-TB) have led to its high mortality worldwide. Relevant data about mortality of XDR-TB patients in literature are limited and likely underestimate the real situation in China, since the majority of patients with XDR-TB are lost to follow-up after discharge from TB hospitals. In this study, we sought to investigate the mortality and associated risk factors of Human Immunodeficiency Virus (HIV)-negative patients with XDR-TB in China.
\end{abstract}

Methods: All patients who were diagnosed with XDR-TB for the first time in four TB care centers across China between March 2013 and February 2015 were consecutively enrolled. Active tracking through contacting patients or family members by phone or home visit was conducted to obtain patients' survival information by February 2017. Multivariable Cox regression models were used to evaluate factors associated with mortality.

Results: Among 67 patients enrolled, the mean age was 48.7 (Standard Deviation [SD] = 16.7) years, and 51 (76\%) were men. Fourteen patients (21\%) were treatment naïve at diagnosis indicating primary transmission. 58 (86.8\%) patients remained positive for sputum smear or culture when discharged. During a median follow-up period of 32 months, 20 deaths occurred, with an overall mortality of 128 per 1000 person-years. Among patients who were dead, the median survival was 5.4 months (interquartile range [IQR]: 2.2-17.8). Seventeen (85\%) of them died at home, among whom the median interval from discharge to death was 8.4 months (IQR: 2.0-18.2). In Cox proportional hazards regression models, body mass index $(\mathrm{BMI})<18.5 \mathrm{~kg} / \mathrm{m}^{2}$ (adjusted hazard ratio [aHR] = 4.5, 95\% confidence interval [Cl]: 1.3-15.7), smoking (aHR $=4.7,95 \% \mathrm{Cl}: 1.7-13.2$ ), or a clinically significant comorbidity including heart, lung, liver, or renal disorders or autoimmune diseases (aHR $=3.5,95 \% \mathrm{Cl}:$ 1.3-9.4), were factors independently associated with increased mortality.

Conclusion: Our study suggested an alarming situation of XDR-TB patients in China with a sizable proportion of newly transmitted cases, a high mortality rate, and a long period in community. This observation calls for urgent actions to improve XDR-TB case management in China, including providing regimens with high chances of cure and palliative care, and enhanced infection control measures.

Keywords: XDR-TB, Mortality, Survival analysis, Risk factors, New case, Transmission

\footnotetext{
* Correspondence: 345480181@qq.com

${ }^{1}$ Changsha Central Hospital, Changsha, Hunan, China

Full list of author information is available at the end of the article
}

(c) The Author(s). 2018 Open Access This article is distributed under the terms of the Creative Commons Attribution 4.0 International License (http://creativecommons.org/licenses/by/4.0/), which permits unrestricted use, distribution, and reproduction in any medium, provided you give appropriate credit to the original author(s) and the source, provide a link to the Creative Commons license, and indicate if changes were made. The Creative Commons Public Domain Dedication waiver (http://creativecommons.org/publicdomain/zero/1.0/) applies to the data made available in this article, unless otherwise stated. 


\section{Background}

Tuberculosis (TB) is one of the top 10 causes of human death [1]. Drug-resistant (DR) TB poses a particular challenge, in which extensively drug-resistant tuberculosis (XDR-TB) is the most severe form. Based on 2017 Global Tuberculosis Report [1], more than 100 countries reported cases of XDR-TB in 2016, the rate of treatment success of XDR-TB were reported merely 30\% in 2014. Although 89 territories and countries had started using bedaquiline and 54 had used delamanid by June 2017 to improve outcomes for Multi-drug resistant tuberculosis (MDR-TB) /XDR-TB, the mortality of XDR-TB reported from treatment cohorts were still above $30 \%$ [1-5]. The high treatment-associated costs are unsustainable in the middle-income and low-income countries that account for over $95 \%$ of tuberculosis related deaths [6]. Meanwhile the "transmitted resistance"-XDR-TB has also well described and confirmed throughout the world $[7,8]$.

China has the third largest TB burden in the world $[1,9]$. In 2016, 895,000 TB cases were newly diagnosed, representing $16 \%$ of new cases worldwide. The prevalence of MDR-TB is $6.6 \%$ among Chinese patients with TB. In addition, based on a 2007 national survey, around $8 \%$ of MDR-TB cases were XDR-TB [10]. Survival situation of Chinese patients with XDR-TB is a crucial parameter to understand the related disease burden. However, relevant data in the literature are limited and likely underestimate the real situation. A multicenter survey in China found that XDR-TB mortality was very low $(4.73 \%, 8 / 169)$ [11]; another study in Shanghai also found that the mortality rate of XDR-TB was in a low level (5.3\%), and $7.4 \%$ was defaulted within $2 \sim 3$ years' treatment [12]. In addition to the lower incidence of Human Immunodeficiency Virus (HIV)-TB coinfections in China, We assume that the main reason for potential under-reported death for XDR-TB in China is the majority of patients with XDR-TB are lost to follow-up after discharge from TB hospitals. Since China had started using bedaquiline for MDR/XDR-TB patients until February 2018, many patients discharged from hospital while still sputum smear/culture positive because of limited therapeutic options and the high treatment-associated costs. For instance, Cui $\mathrm{H}$ et al. reported that $50 \%$ of patients with XDR-TB were not traceable after discharge from a TB referral hospital in Beijing [3]. Mortality of Chinese patients with XDR-TB has not been assessed using active tracking to address the issue of losing patients to follow-up.

With this study, we sought to evaluate the mortality of HIV-negative patients with XDR-TB and its related influencing factors through active tracking in four TB specialized hospitals in China.

\section{Methods}

Patients enrollment

All patients admitted into four TB specialist hospitals (Changsha Central Hospital, Wuhan Treatment Center, the Third People's Hospital of Hengyang, and the Second People's Hospital of Chenzhou) and initially diagnosed as XDR-TB by drug susceptibility testing (DST) between March 2013 and February 2015 were included into this study. Patients with previous confirmed XDR-TB and/or HIV-positive were excluded.

\section{Ethical approval}

All patients' information was routinely recorded and collected by attending physicians. This study was approved by the Ethical Review Committee of responsible units (Changsha Central Hospital) and we had received ethics committee approval for verbal consent. We obtained verbal informed consent from all subjects on the phone, if the patient died when we tracked, the verbal consent was received from the legal representative or next of kin of the deceased patients.

\section{Tracking and data collection}

After the patients enrollment, Demographic and clinical information (including name, sex, age, height, weight, telephone and address, marriage, occupation, smoking history, history of drinking, tuberculosis course and treatment history, situation on combing with underlying diseases, hemoglobin, serum albumin) were obtained from medical record review. Effort was made to assess the vital status online for all patients with XDR-TB until February 2017. For the cases medical records not comprehensive, a phone interview or home visit was conducted. The end time of tracking of the patients who survived was February 16, 2017, and the death time of the dead patients. The end events of tracking was death of all deaths. The patient enrollment and tracked process is illustrated in Fig. 1.

\section{Definition}

XDR-TB was defined by resistance to isoniazid, rifampicin, and at least one injectable agent (i.e., amikacin, kanamycin, capreomycin) and any of the fluoroquinolones $[13,14]$. New TB cases were TB patients who had denied having any previous anti-TB treatment or history of more than 30 days of anti-TB treatment. Retreatment TB cases were defined as TB patients who had been accepting TB treatment for more than 30 days or who had recorded evidence of prior treatment in the surveillance database or case reports $[15,16]$.

\section{Statistical analysis}

The baseline clinical features of XDR-TB patients were analyzed by descriptive analysis. Cox proportional 
72 patients with XDR-TB were initially diagnosed

in four TB specialized hospitals between March 2013 and February 2015.

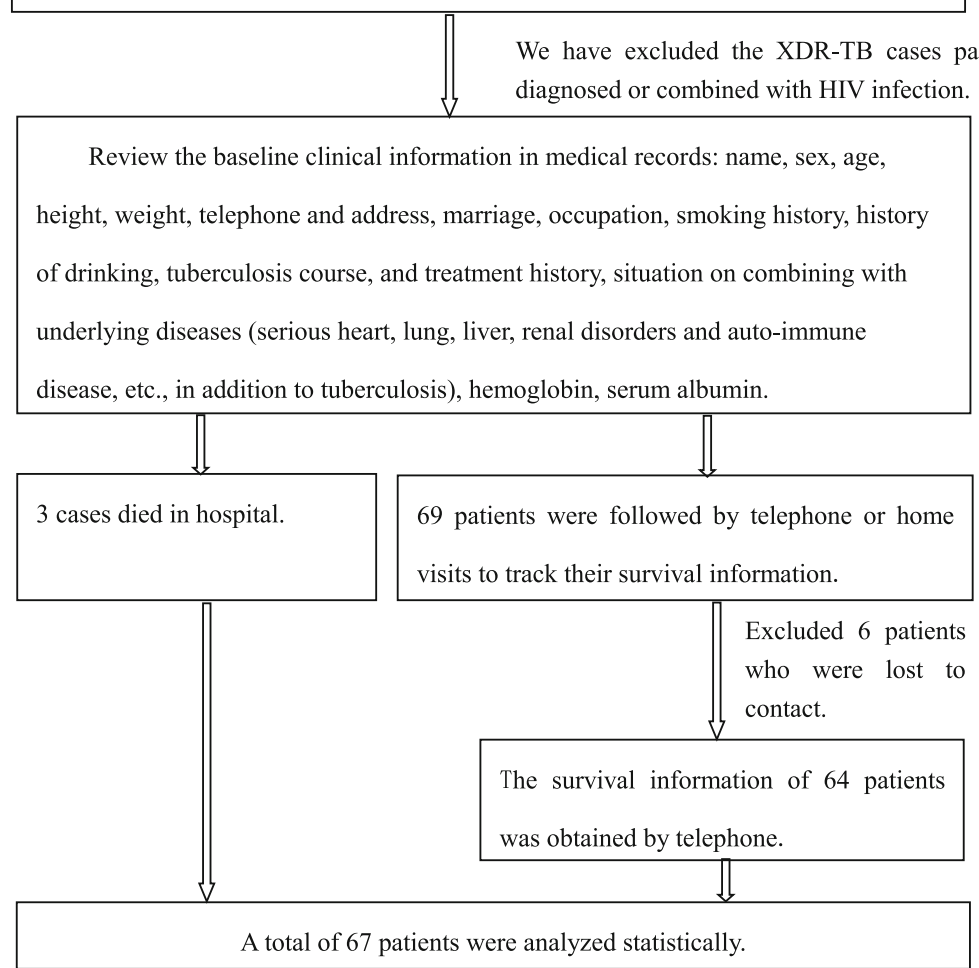

Fig. 1 The enrollment and tracked process of study population of XDR-TB cases in four TB specialized hospitals, March 2013 February 2015

hazards regression model was used to evaluate the association between baseline laboratory and clinical variables and death. The method used was "Backward:LR"; the probability stepwise was "entry $=0.05$ " and "removal = 0.1." The Kaplan-Meier method was used to map the survival curves. SPSS software (version 22.0) was used to perform all statistical analyses, with a " $P<0.05$ " as the criterion for indicating statistical significance.

\section{Results}

\section{Characteristics of enrolled patients with XDR-TB}

Average age at admission was $(48.7 \pm 16.7)$ years (range, 19-99 years), and the average body mass index (BMI) was $18.9 \pm 3.7 \mathrm{~kg} / \mathrm{m}^{2}$ (range, $13.4-30.7 \mathrm{~kg} / \mathrm{m}^{2}$ ), $51(76 \%)$ patients were men, and $42(63 \%)$ were off-site-referral patients. Of all patients, 79\% (53/67) had a history of TB treatment, and the remaining $21 \%(14 / 67)$ were new TB cases.

\section{Outcomes of XDR-TB patients}

Among the 67 patients, 3 patients died during hospitalization, 6 patients discharged from the hospital while sputum smear/culture converted to negative, and $58(86.8 \%)$ patients remained positive for sputum smear or culture when discharged. A phone interview was conducted on the 64 patients who were still alive at discharge in February 2017.

Until February 16, 2017, the median follow-up time was 32 (interquartile range [IQR]: 20.3-38.4) months (range, 0.7-47.1 years). During the tracked period, 20 patients died. The total annual mortality rate was $12.8 \%$. Among patients who were dead, the median survival was 5.4 months (IQR: 2.2-17.8). In patients who died during the tracked period, the median survival was only 5.4 (IQR: 2.2-17.8) months; three of them died in the hospital, 17 (85\%) died at home, Seventeen $(85 \%)$ of them died at home, among whom the median interval from discharge to death was 8.4 months (IQR: 2.0-18.2) and the median time from discharge to death was 8.4 (IQR: 2.0-18.2) months. The cumulative survival curve (Fig. 2) showed that 14 patients died in the first year, accounting for $70 \%$ of all deaths, and 10 died in the first 6 months, accounting for $50 \%$ of all deaths.

Among the 47 survivors, 9 cases said they had been cured, 17 cases were continuing treatment with some "old and less active" anti TB drugs, the other 21 cases had given up treatment or just accepted palliative care such as nutritional support and cough relieving, only 12 


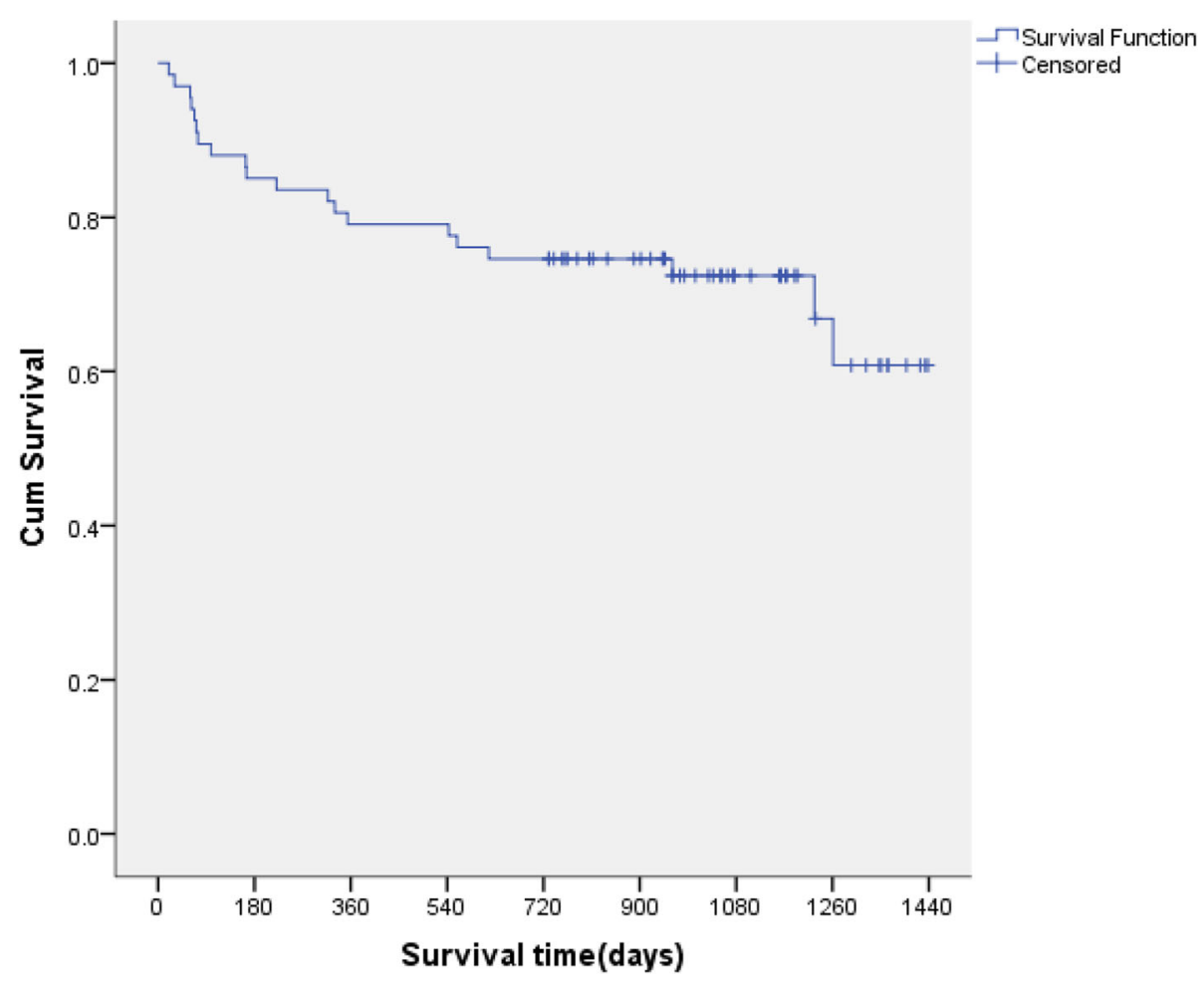

Fig. 2 The survival curve of 67 XDR-TB patients

of them said they visited the hospital regularly for examination and follow-up.

\section{Mortality associated factors}

In univariable models, age $>50$ years, history of smoking, $\mathrm{BMI}<18.5 \mathrm{~kg} / \mathrm{m}^{2}$, combined with the underlying disease, hemoglobin $(\mathrm{Hb})$ concentration $<115 \mathrm{~g} / \mathrm{L}$, and albumin $(\mathrm{ALB})<35 \mathrm{~g} / \mathrm{L}$ at admission were associated with increased risk of death. In multivariable models, lower BMI (adjusted hazard ration $[\mathrm{aHR}]=4.5$, 95\% confidence interval [CI]: 1.3-15.7), history of smoking ( $\mathrm{aHR}=4.7 ; 95 \% \mathrm{CI}: 1.7-13.2)$, and combined with the underlying disease $(\mathrm{aHR}=3.5 ; 95 \% \mathrm{CI}$ : 1.3-9.4) remained statistically significant (Table 1 ).

\section{Discussion}

In our study, A total of 67 patients from four large-scale Tuberculosis Specialized Hospitals were first confirmed

Table 1 Survival Analysis of 67 XDR-TB Patients

\begin{tabular}{|c|c|c|c|c|c|}
\hline \multirow[t]{2}{*}{ Variables } & \multirow{2}{*}{$\begin{array}{l}\text { No. of } \\
\text { cases, } \\
\text { n (\%) }\end{array}$} & \multicolumn{2}{|c|}{ Univariate Analysis } & \multicolumn{2}{|c|}{ Multivariate Analysis } \\
\hline & & $\mathrm{HR}(95 \% \mathrm{Cl})$ & $P$ & aHR $(95 \% C l)$ & $P$ \\
\hline Age $>50$ years & $32(47.8)$ & $2.82(1.08-7.35)$ & 0.034 & $2.40(0.84-6.85)$ & 0.103 \\
\hline Male sex & $51(76.1)$ & 1.32(0.44-3.96) & 0.619 & - & \\
\hline $\mathrm{BMl}<18.5 \mathrm{~kg} / \mathrm{m}^{2}$ & $40(59.7)$ & $4.30(1.26-14.72)$ & 0.020 & $4.52(1.31-15.65)$ & 0.017 \\
\hline $\mathrm{Hb}<115 \mathrm{~g} / \mathrm{L}$ & 19(28.4) & $2.49(1.03-6.03)$ & 0.043 & $2.08(0.83-5.20)$ & 0.117 \\
\hline$A L B<35 \mathrm{~g} / \mathrm{L}$ & 24(35.8) & $4.37(1.73-11.03)$ & 0.002 & $1.07(0.27-4.20)$ & 0.920 \\
\hline TB course $>3$ years & $32(47.8)$ & $1.51(0.62-3.68)$ & 0.369 & - & \\
\hline Retreatment TB cases & $53(79.1)$ & $0.43(0.17-1.08)$ & 0.073 & & \\
\hline Smoking history & $27(40.3)$ & $3.83(1.51-9.73)$ & 0.005 & $4.67(1.66-13.16)$ & 0.004 \\
\hline Drinking history & 10(14.9) & $2.18(0.79-6.01)$ & 0.132 & & \\
\hline combined with underlying diseases & $23(34.3)$ & $5.16(2.05-13.00)$ & 0.001 & $3.48(1.30-9.36)$ & 0.013 \\
\hline
\end{tabular}

Definition of abbreviations: $X D R$ extensive drug-resistant, $T B$ tuberculosis, $H R$ hazard ration, $C I$ confidence interval, aHR adjusted hazard ration, $B M I$ body mass index, $H b$ hemoglobin, $A L B$ albumin 
as XDR-TB cases and tracked for a median follow-up period of 32(IQR: 20.3-38.4) months. The data showed an overall mortality of 128 per 1000 person-year, and the median survival of deaths was only 5.4 (IQR: 2.2-17.8) months, which was significantly more serious than that reported in China [11, 12]. Smoking, BMI $<18.5 \mathrm{~kg} / \mathrm{m}^{2}$ and the situation on combining of underlying diseases were significant predictors of mortality in this study. We also found $15 \%$ of deaths $(3 / 20)$ were classified as died in the hospital, other $85 \%$ died at home, and the median interval from discharge to death was 8.4 (IQR: 2.0-18.2) months. In addition, 58(86.8\%) patients remained positive for sputum smear or culture when discharged, indicating potential transition in the community, which was worthy of our further study and research.

To our knowledge, this is the first study in China to evaluate the mortality and survival analysis of inpatients with XDR-TB using active tracking. In this study, $85 \%$ of deaths were captured through telephone interview or home visits rather than medical record review, which supported our speculation that passive collection of vital status information would lead to considerable underestimation of mortality. In our study, 20 deaths occurred among 67 patients enrolled, translated into an overall mortality of 128 per 1000 person-year. The mortality greatly exceeds the relevant data in the previous literature and also exceeds the mortality of total HIV-negative patients with TB in China, which was reported 3.6(2.45) per 100,000 person-year in the 2017 Global Tuberculosis Report [1]. Obviously, survival situation of Chinese patients with XDR-TB is a crucial parameter to understand the related disease burden and calls for urgent actions to improve XDR-TB case management.

Among patients who were dead during tracking period, the median survival time was only 5.4 (IQR: $2.2-$ 17.8) months, The findings suggested an alarming situation of XDR-TB patients in China. The cumulative survival curve showed that 14 patients died in the first year, accounting for $70 \%$ of all deaths, and 10 died in the first 6 months, accounting for $50 \%$ of all deaths. The data are similar to those reported in South Africa [17] and suggests that the first year (especially the first half year) after initial diagnosis of XDR-TB was responsible for the high risk of death. Patients in this period must be fully treated and given adequate attention to survive the high-risk period and win the time for the following treatment. Fan L et al. [5] found 49 deaths in 107 patients with XDR-TB in three provinces of South Africa after the follow-up of 24 months; the mortality rate (46\%) was significantly higher than that in our study. Sheela [18] reported that the median survival (34 days [IQR: 18-90]) of XDR-TB patients who died within 180 days were also significantly shorter than that in our study. Excluding the lower living and medical conditions and other factors, we considered that the group of patients in our study ruled out the case of XDR-TB combined with HIV infection, which may greatly raise the mortality rate. It had been proven long ago that $\mathrm{TB}$ and HIV co-infection in South Africa was more than $70 \%$, and XDR-TB combined with HIV patients had a long-term survival rate of less than $20 \%[6,7]$.

Baseline clinical variables, including BMI $<18.5 \mathrm{~kg} / \mathrm{m}^{2}$ and the combination of underlying diseases, were significant predictors of mortality in this study. Our findings are in line with those of a study from Korea in which XDR-TB patients with BMI $<18.5 \mathrm{~kg} / \mathrm{m}^{2}$ had higher mortality [19]. Tang et al. [11] also demonstrated that BMI $<18.5 \mathrm{~kg} / \mathrm{m}^{2}$ or comorbidities were an important factor in the poor prognosis of MDR-TB and XDR-TB. The implication of these findings is that patients with risk indicators should be given specific attention, and supportive intervention should be considered. We also found that XDR-TB patients with smoking history had higher mortality. The finding is potentially significant due to the high smoking rate in China and other countries. According to a territory-wide treatment program in Hong Kong, [20] smoking contributed to $16.7 \%$ of unsuccessful treatment outcomes after 2 years of follow-up and affected baseline disease severity, relapse, and bacteriologic response. As a result, smoking should be forbidden in DR-TB patients, especially in XDR-TB groups.

The study found that $63 \%$ of the XDR-TB patients were referred from off-site, $85 \%$ returned to the community, only 47 patients (70\%) survived. Among the 47 survivors, only 12 patients said they visited the hospital regularly for examination and follow-up, we therefore infered that other patients did not accept DOT (Directly Observed Treatment, DOT) and standardized management at home. As expected, the study found that majority of the survivors only accepted some "old and less active" anti TB drugs or palliative care or even gave up treatment after being diagnosed as XDR-TB because of lack of effective therapeutic agents and methods, or economic difficulties, etc. The data from our tracking show that these $85 \%$ of patients survived in the community for 8.4 (IQR: 2.0-18.2) months, and in the course of 8.4 months, they lacked strong medical treatment conditions, awareness, and management conditions to control transmission in families and communities. So, the resistant strains can be transmitted repeatedly and widely in their daily activities, as well as in the process of the medical treatment and referral. These findings have brought great risks and challenges to our intervention and management, [21] and calls for rapid roll out of and access to the new drugs. The study also found that $21 \%$ of XDR-TB patients were new cases, who had denied having any previous anti-TB treatment and could be diagnosed as "transmitted resistance" only by reviewing the 
medical records. This result suggested that the spread of XDR-TB in China cannot be ignored. At present, the spread of MDR-TB and XDR-TB strains is a serious problem all around the world [22, 23]. Although the proportion of "transmitted resistance" in this study was significantly lower than that reported by South Africa (69.3\%) [17] and Shanghai $(54.5 \%)$ [24]. We anticipate that some patients who were diagnosed as "acquired resistance" by reviewing the history of anti-TB treatment in medical records might be un-detected DR-TB patients due to unavailability of DST upon the point when they were first diagnosed as TB and provided with anti-TB treatment for susceptible TB patients. We therefore reinforce the importance of universal access of DST for both new and retreatment TB cases, which is already stated in China National TB Program (NTP). Although there is no difference on treatment strategy for transmitted versus acquired XDR-TB, and no significant difference in mortality and survival between the two ( $\mathrm{HR}=0.43,95 \% \mathrm{CI}$ : $0.17-1.08, P=0.073)$, interventions for preventing transmitted versus acquired disease differ. Acquired drug resistance can be decreased by ensuring completion of treatment and providing effective treatment. Stopping transmission requires determining and separating infectious patients, promptly initiating effective treatment, and improving ventilation in congregate settings. Therefore, the effective management of drug-resistant patients and regular treatment are equally important.

There are some limitations to this study. First, because patients were enrolled from four TB care centers, caution should be taken when the findings of this study are extrapolated into another setting in China. The resistance prevalence observed is likely higher than the nationwide average, representing a more serious situation. Second, the study included patients admitted between March 2013 and February 2015. Some patients were followed for only 2 years, and the time for observing the outcome was possibly short. Third, the study found that $21 \%$ of XDR-TB patients were new cases and the other $79 \%$ of the patients went to the referral hospital on a relatively advanced stage of the tuberculosis, which may lead to underestimation of survival time. In addition, Some studies, we compared mortality with which even in China or South Africa, were reported mortality during treatment cohort, while partial XDR-TB patients in our study were untreated, which could lead to overestimate the real mortality than the treatment cohort. But since XDR-TB lacks effective therapeutic regimens and drugs worldwide currently, we think the ratio of overvaluation will not be obvious.

\section{Conclusions}

Our study suggested an alarming situation of XDR-TB patients in China with a high mortality rate, a sizable proportion of newly transmitted cases, and a long period in community. This observation calls for urgent actions in China to improve XDR-TB case management in China, including providing regimens with high chances of cure and palliative care, and enhanced infection control measures. In addition, XDR-TB patients with BMI < $18.5 \mathrm{~kg} / \mathrm{m}^{2}$, combined with underlying diseases and smoking history, were significant predictors of mortality and should be given special attention.

\begin{abstract}
Abbreviations
aHR: Adjusted hazard ration; ALB: Albumin; BMl: Body mass index; Cl: Confidence interval; DOT: Directly observed treatment; DR: Drug-resistant; DST: Drug susceptibility testing; Hb: Hemoglobin; HIV: Human Immunodeficiency Virus; HR: Hazard ration; IQR: Interquartile range; MDR: Multi-drug resistant; NTP: National TB Program; SD: Standard deviation; TB: Tuberculosis; XDR: Extensive drug-resistant

\section{Funding}

The study was provided by The National Major and Technological Special Project for infectious disease during the Twelfth Five-year Plan Period (grant No. 2013ZX10005004), and China Tuberculosis Clinical Trial Consortium (CTCT C) research funding (grant No. 2017KYJJ008). The funders contributed to the study design and data collection.
\end{abstract}

\section{Availability of data and materials}

The datasets supporting the conclusions of this article was presented within the manuscript.

\section{Authors' contributions \\ Conceived and designed the study: CB, MF, YZ, KY, YhL, YKL, LY. Collected data, tracked with patients, analyzed the data and interpreted the results: $C B$, $H X, L Z, B X, F L, H H, Y K L, J Z$. Wrote the paper: CB. Revised the manuscript: CB, $Y Z, Y h L, H X, L Z, B X, F L, H H, J Z, M F, K Y, Y K L, L Y$. All authors have read and approved the final manuscript.}

Ethics approval and consent to participate

This study was approved by the Ethical Review Committee of responsible units (Changsha Central Hospital) and we had received ethics committee approval for verbal consent. We obtained verbal informed consent from all subjects on the phone, if the patient died when we tracked, the verbal consent was received from the legal representative or next of kin of the deceased patients.

\section{Competing interests}

The authors declare that they have no competing interests.

\section{Publisher's Note}

Springer Nature remains neutral with regard to jurisdictional claims in published maps and institutional affiliations.

\section{Author details \\ ${ }^{1}$ Changsha Central Hospital, Changsha, Hunan, China. ${ }^{2}$ Beijing Innovation Alliance of TB Diagnosis and Treatment, Beijing, China. ${ }^{3}$ Wuhan Medical Treatment Center, Wuhan, Hubei, China. ${ }^{4}$ The Third People's Hospital of Hengyang, Hengyang, Hunan, China. ${ }^{5}$ The Second People's Hospital of Chenzhou, Chenzhou, Hunan, China. ${ }^{6}$ China Center on TB, China CDC, Beijing, China.}

Received: 11 January 2018 Accepted: 28 May 2018

Published online: 07 June 2018

\section{References}

1. World Health Organization. Global tuberculosis report 2017. WHO/HTM/TB/ 2017.23. Geneva: World Health Organization. 2017. p. 21-63.

2. Kvasnovsky CL, Peter CJ, Van dWML. Treatment outcomes for patients with extensively drug-resistant tuberculosis, KwaZulu-Natal and Eastern Cape Provinces, South Africa. Emerg Infect Dis. 2016;22(9):1529-36. 
3. Liu CH, Li L, Chen Z, et al. Characteristics and treatment outcomes of patients with MDR and XDR tuberculosis in a TB referral hospital in Beijing: a 13-year experience. PLoS One. 2012;6(4):e19399.

4. Falzon D, Gandhi N, Migliori GB, et al. Resistance to fluoroquinolones and second-line injectable drugs: impact on multidrug-resistant TB outcomes. Eur Respir J. 2013;42(1):156-68.

5. Fan L, Sha W. Long - term prognosis of extensively drug-resistant TB (XDRTB) in South Africa: a cohort study. Chin J Tuberc Res Dis. 2014;37(10):800.

6. Pietersen E, Ignatius E, Streicher EM, et al. Long-term outcomes of patients with extensively drug-resistant tuberculosis in South Africa: a cohort study. [J]. Lancet. 2014;383(9924):1230-9.

7. Gandhi NR, Moll A, Sturm AW, et al. Extensively drug-resistant tuberculosis as a cause of death in patients co-infected with tuberculosis and HIV in a rural area of South Africa. Lancet. 2006;368(9547):1575-80.

8. Wells CD, Cegielski JP, Nelson $\sqcup$, et al. HIV infection and multidrug-resistant tuberculosis—-the perfect storm. J Infect Dis. 2007;196(Suppl 1(2)):S86-107.

9. Organization W H. Multidrug and extensively drug-resistant TB (M/XDR-TB): 2010 global report on surveillance and response. World Health Organization. 2010;35(6):2171-8.

10. Espinal MA, Laserson K, Camacho M. National Survey of drug-resistant tuberculosis in China. New Engl J Med. 2012;366(23):2161-70.

11. Tang S, Tan S, Yao L, et al. Risk factors for poor treatment outcomes in patients with MDR-TB and XDR-TB in China: retrospective multi-center investigation. PLoS One. 2013;8(12):e82943.

12. Tang SJ, Zhang Q, Xiao HP. Etal. Extensively drug-resistant tuberculosis at a tuberculosis specialist hospital in shanghai, China: clinical characteristics and treatment outcomes. Scand J Infect Dis. 2011;43:280-5.

13. Organization WH. Companion Handbook to the WHO Guidelines for the Programmatic Management of Drug-Resistant Tuberculosis. World Health Organization. 2014;14(6):672-82.

14. China Anti - tuberculosis Association. Guidelines for Chemotherapy for Drug - Resistant Tuberculosis (2015). Chin J Antituberc. 2015;37(5):421-69.

15. Falzon D, Jaramillo E, Schünemann H. The 2011 update of the World Health Organization guidelines for the programmatic management of drugresistant tuberculosis. Eur Respir J. 2011;38(3):516-28.

16. Organization WH. Management of MDR-TB: a field guide: a companion document to guidelines for Programmatic Management of Drug-Resistant Tuberculosis: Integrated Management of Adolescent and Adult IIIness (IMAI). Pearson Schweiz Ag. 2009;63(3):307-19.

17. Shah NS, Auld SC, Brust JC, et al. Transmission of extensively drug-resistant tuberculosis in South Africa. N Engl J Med. 2017;376(3):243-53.

18. Shenoi SV, Brooks RP, Barbour R, et al. Survival from XDR-TB is associated with modifiable clinical characteristics in rural South Africa. PLoS One. 2012; 7(3):e31786.

19. Kim DH, Kim HJ, Park SK, et al. Treatment outcomes and survival based on drug resistance patterns in multidrug-resistant tuberculosis. Am J Respir Crit Care Med. 2010;182(1):113-9.

20. Leung CC, Yew WW, Chan CK, et al. Smoking adversely affects treatment response, outcome and relapse in tuberculosis. Eur Respir J. 2014;45(3):738-45.

21. Sharma V, Aggarwal S. XDR tuberculosis: a looming threat. Indian J Community Med. 2009;34(2):166.

22. Farley JE, Tudor C, Mphahlele M, et al. A national infection control evaluation of drug-resistant tuberculosis hospitals in South Africa [J]. Int J Tuberc Lung Dis. 2012;16(1):82-9.

23. Tudor C, Walt MVD, Hill MN, et al. Occupational health policies and practices related to tuberculosis in health care workers in KwaZulu-Natal, South Africa. Public Health Action. 2013;3(2):141-5.

24. Ming Z, Xia L, Peng X, et al. Transmission of MDR and XDR tuberculosis in shanghai, China. PLoS One. 2009;4(2):e4370.

\section{Ready to submit your research? Choose BMC and benefit from:}

- fast, convenient online submission

- thorough peer review by experienced researchers in your field

- rapid publication on acceptance

- support for research data, including large and complex data types

- gold Open Access which fosters wider collaboration and increased citations

- maximum visibility for your research: over $100 \mathrm{M}$ website views per year

At BMC, research is always in progress.

Learn more biomedcentral.com/submissions 